(4) Gli articoli di questa sezione sono sottoposti a referaggio doppiamente cieco (double blind peer review process) e seguono gli standard in uso per le pubblicazioni scientifiche a livello internazionale ed accettati dalle principali banche dati citazionali

\title{
Anziani e gioco d'azzardo: una ricerca su consapevolezza, abitudini e rischi tra gli over 65 anni con il Senior Problem Gambling Questionnaire (SPGQ)
}

\author{
Annalisa Pistuddi*, Paola Broggi ${ }^{\circ}$, Marco Cavicchioli ${ }^{\circ}$, Alfio Lucchini $^{\S}$
}

\begin{abstract}
SUMMARY
- The empirical research has recently focused attention on pathological and non pathological gambling among elderly population, showing a complex phenomenon sustained by several aspects which include sociological, psychological and biological determinants. Nevertheless, few studies in literature explore gambling habits in an Italian population over 65 years of age. In line with the previous aim, an author of the current work built a 9item self-report instrument called Senior Problem Gambling Questionnaire (SPGQ).SPGQ mainly explores two different aspects of gambling in elderly population: a) level of awareness related to negative consequences associated with gambling; $b$ ) severity of gambling behaviors. SPGQ was administered to 387 subjects. 353 individuals completed the screening evaluation. Gender was homogeneously distributed among subjects. $85.6 \%$ of sample showed good level of negative consequences awareness associated with gambling, 22.2\% of subjects affirmed to engage in gambling behaviors at least once a week, $4.4 \%$ of individuals affirmatively answered to both pathological gambling items. Although men seemed to engage in gambling behaviors more frequently than women, gender was homogeneously distributed among individuals who engage in gambling behaviors at least two times a week. Eventually, this is the first study that shows a modest albeit epidemiologic relevant portion of Italian individuals over 65 years of age which might be pathological gamblers.
\end{abstract}

Keywords: Gambling, Older adults, SPGQ, Awareness, Risk, Problematic behavior.

Parole chiave: Gioco d'azzardo, Anziani, SPGQ, Rischio, Comportamento problematico.

\section{Introduzione}

Il fenomeno del gioco d'azzardo patologico sta destando interesse crescente in ambito sanitario, sia in termini di attenzione della ricerca medico-scientifica che in termini di politiche nel campo della prevenzione e del trattamento.

\footnotetext{
* Psicologa psicoterapeuta, sessuologa, consulente del Dipartimento delle Dipendenze ASST Melegnano e della Martesana. E-mail: annalisa.pistuddi@asst-melegnano-martesana.it.

- Psicologa psicoterapeuta, specialista in psicologia clinica, consulente del Dipartimento delle Dipendenze ASST Melegnano e della Martesana e del Servizio Alcoldipendenze e Psicologia Clinica Ospedale San Raffaele Turro - Milano.E-mail: paola.broggi@asst-melegnano-martesana.it.

${ }^{\circ}$ Psicologo, consulente del Servizio Alcoldipendenze e Psicologia Clinica Ospedale San Raffaele Turro - Milano/Università Vita-Salute San Raffaele.E-mail: cavicchioli.marco@hsr.it.

\& Psichiatra, specialista in psicologia medica, psicoterapeuta, direttore del Dipartimento delle Dipendenze e di Salute Mentale ASST Melegnano e della Martesana, professore a.c. Università Cattolica Milano.Past President di FeDerSerD.E-mail: alfio.lucchini@asstmelegnano-martesana.it.
}

Sebbene il gioco d'azzardo patologico possa coinvolgere persone di qualunque fascia di età, quella degli over 65 anni appare particolarmente vulnerabile e rappresenta al contempo un target interessante e specifico per le industrie del gioco (Alberghetti, 2015). Ciononostante l'impatto del gioco d'azzardo sulla popolazione anziana, sia a livello ricreativo che patologico, è a tutt'oggi poco esaminato ed è ancora bassa l'attenzione della ricerca sulle caratteristiche dei giocatori d'azzardo anziani, sulle loro modalità di gioco, sulle loro motivazioni al giocare d'azzardo, sui fattori specifici che li distinguono da altre fasce di età. Alcuni autori ad esempio (Mc Neilly, 2002; Guiducci, 2013) hanno posto l'accento su fattori specifici di questa fase del ciclo vitale: la quotidianità, il tempo libero in eccesso, le poche attività ricreative, la tendenza all'isolamento e la disponibilità finanziaria costante derivante dalla pensione o da una rendita, rappresentano elementi che possono favorire il comportamento di gioco. La perdita dell'identità sociale può favorire condizioni di solitudine e di isolamento rispetto alla situazione di vita lavorativa e familiare del passato; questi vissuti possono creare terreno fertile per la ricerca di abitudini ed attività quali il gioco d'azzardo, volte a socializzare ma anche a sottrarsi o ad alleviare eventuali condizioni negative e spiacevoli (McNeilly, 2000; Pavalko, 2002). II concorrere di questi elementi può rendere ancora più 
difficile intercettare una condotta patologica di gioco nell'anziano. Proprio a questo riguardo Desai (2004) rileva una differenza tra giocatori d'azzardo adulti e anziani: da una parte le perdite finanziarie per un anziano possono essere quantitativamente più cospicue, dall'altra l'impatto sulle relazioni familiari può essere smorzato, se non assente, rispetto alla situazione degli adulti lavoratori.

Anche in Italia scarseggiano le ricerche condotte sul fenomeno del gioco d'azzardo nella popolazione anziana, sebbene l'interesse stia crescendo. Ad esempio la Lombardia, tra le regioni che maggiormente hanno recepito la normativa nazionale (Decreto Balduzzi), è una regione pilota in merito all'applicazione di normative specifiche (Legge n. 8/2013 e seguenti); inoltre alcuni distretti lombardi hanno focalizzato la loro attenzione coinvolgendo la cittadinanza in momenti di confronto sul tema del gioco d'azzardo e alla popolazione anziana sono stati rivolti interventi informativi specifici. Un'interessante ricerca svolta sulla popolazione bergamasca è stata promossa recentemente dal Dipartimento delle Dipendenze della ASL di Bergamo (Beato 2015), in collaborazione con Federconsumatori, Adiconsum, Sindacati Pensionati CGIL e CISL, Consiglio di Rappresentanza dei Sindaci, L'Eco di Bergamo. Condotta dal CNR, costituisce il primo studio statistico sulla popolazione anziana a livello italiano. Si è attuato inviando per posta un questionario a tutti i residenti della provincia di Bergamo di un'età compresa tra i 65 e 84 anni: 2100 sono stati i questionari debitamente compilati e quindi ritenuti validi. Dai dati rilevati è emerso che il $41 \%$ degli anziani pratica un gioco d'azzardo non problematico, a connotazione ricreativa e sociale, mentre $\mathrm{I}^{\prime} 8,7 \%$ mostra un gioco definito a rischio o problematico, pari al $17,7 \%$ del campione dei giocatori. Le caratteristiche di questa condotta di gioco sono definibili con la tendenza a giocare quantità crescenti di denaro, con la tendenza a giocare nuovamente per tentare di recuperare il denaro perduto, con la perdita di autocontrollo sul comportamento di gioco.

Vista la carenza di studi scientifici sulla popolazione anziana italiana a proposito delle abitudini nei confronti del gioco d'azzardo e del rischio di sviluppare una condotta problematica, la Task Force della ex-ASL Milano 2 ha creato un questionario di semplice somministrazione, volto alla rilevazione di specifici aspetti associati al gioco d'azzardo, che potesse essere impiegato all'interno di azioni di sensibilizzazione sul territorio della attuale ASST Melegnano e della Martesana. Gli incontri sono stati organizzati con le persone con più di 65 anni, in collaborazione con alcuni Comuni ed associazioni del privato sociale e condotti dal personale del Task Force GAP della ex-ASL Milano 2; questo momento organizzativo è stato confermato dalla ASST.

\section{Presentazione del questionario Senior Problem Gambling Que- stionnaire (SPGQ)}

Il questionario è denominato Senior Problem Gambling Questionnaire (SPGQ) ed è stato ideato da Annalisa Pistuddi, psicologa della ASST Melegnano e della Martesana, in collaborazione con il Gruppo Italiano per lo Studio dello Stress e delle Metodiche Antistress (GISSMA).

La creazione del SPGQ è finalizzata a tre scopi: condurre una rilevazione epidemiologica del fenomeno del gioco d'azzardo sulla popolazione anziana, colmando la carenza degli strumenti ad oggi disponibili; evidenziare possibili condizioni di problematicità nel gioco d'azzardo tra gli anziani sul territorio lombardo; in ultimo, trasmettere l'attenzione che gli operatori dei servizi sociali pongono alle fragilità della popolazione anziana.

Il questionario è rivolto alle persone con almeno 65 anni di età, è anonimo e può essere impiegato in forma auto o etero-somministrata. II questionario è stato costruito utilizzando items di tipo dicotomico al fine di semplificare la sua compilazione, tenendo in considerazione la popolazione over 65 anni di età a cui è rivolto. La strutturazione dei quesiti del questionario ha tenuto in considerazione principalmente due aspetti che sono considerati rilevanti al fine di identificare il rischio di sviluppo di problemi nelle condotte di gioco d'azzardo: a) la conoscenza/consapevolezza del fenomeno del gioco d'azzardo; b) la rilevazione di comportamenti associati al gioco d'azzardo problematico.

In linea con la popolazione di riferimento e con l'obiettivo di facilitare la sua compilazione in tempi rapidi, si è scelto di proporre una versione dello strumento costituito da 9 items che potessero equamente indagare i due aspetti precedentemente descritti. Nello specifico, gli items 1,2,8,9 sono stati ideati per esplorare la conoscenza e la consapevolezza della problematica del gioco d'azzardo; per contro, gli items 3,4,5,6,7 sono stati delineati con la finalità di rilevare la presenza di comportamenti associati al gioco d'azzardo, e più nello specifico, gli items 6,7 sono connessi con risvolti problematici o patologici.

In aggiunta, data la finalità di rilevazione epidemiologica del fenomeno del gioco d'azzardo attraverso lo strumento in esame, sono stati inclusi due quesiti (associati rispettivamente agli items 3 e 4) volti a raccogliere informazioni circa le tipologie dei giochi che vengono praticate, sia nel periodo attuale che nel passato. Inoltre, è stato inserito un quesito aggiuntivo (item 5) volto all'indagine della frequenza settimanale delle condotte di gioco d'azzardo nel caso esse siano presenti Le informazioni sociodemografiche raccolte sono: il luogo in cui il questionario è stato compilato, l'età del soggetto, il sesso, lo stato civile. Il questionario è allegato in fondo al presente lavoro.

\section{Materiali e metodi}

Il questionario SPGQ è stato somministrato a partire dall'aprile 2015 sino a giugno 2016, nel corso di tutti gli interventi che il personale della Task Force GAP ha rivolto alla popolazione anziana.

In linea con la struttura dicotomica degli items dello strumento, è stata proposta l'analisi delle componenti principali categoriale (CATPCA) al fine di studiare la struttura fattoriale del questionario e la sua consistenza interna ( $\alpha$ di Cronbach).

Il test del $\chi^{2}$ è stato utilizzato per esplorare la relazione esistente tra il genere e le risposte fornite ai vari items dello strumento al fine di indagare possibile differenze nelle caratteristiche associate al gioco d'azzardo tra uomini e donne. Inoltre, le suddette relazioni sono state esplorate all'interno di specifiche modalità di gioco d'azzardo, così come all'interno della frequenza di messa in atto di tali comportamenti. Infine, sono state indagate le differenze di genere in relazione alla compresenza di risposte affermative agli item 6,7 , i quali descrivono le caratteristiche nucleari del GAP.

\section{Risultati}

Il totale dei questionari somministrati è stato di 387, di cui validi (ovvero con non più di una risposta mancante) sono stati 353, pari all'91,2\% del totale. Il test di Little $\left(\chi^{2}(104)=116,82 ; p=\right.$ 
$0,18)$ ha mostrato una distribuzione dei dati mancanti Missing Completely At Random (MCAR). Di conseguenza è possibile concludere come i soggetti con dati mancanti non si discostano significativamente dai soggetti che hanno completato la valutazione.

Il campione ha un'età media di 75,9 anni, con un minimo di 65 anni di età ed un massimo di 98 anni; la deviazione standard è pari a 6,8 anni, il che indica che la maggioranza del campione si colloca tra i 70 e gli 80 anni. Il campione è bilanciato per sesso: il $50,9 \%$ è composto da donne $(\mathrm{N}=173)$ ed il $49,1 \%$ da uomini $(\mathrm{N}=167)$; il 3,7\%, corrispondente a 13 persone, non ha risposto al quesito.

In linea con i principi che hanno guidato la costruzione dello strumento, è stata proposta un'indagine della struttura dello strumento caratterizzata da due dimensioni. I risultati hanno mostrato come la struttura bifattoriale non sia supportata. Nello specifico, la prima dimensione, costituita dagli items $1,3,4,5,6,7,8$, ha mostrato una consistenza interna adeguata $(\alpha=0,77 ; 35,41 \%$ di varianza spiegata); per contro, la seconda dimensione, costituita dagli items 2,9 ha mostrato una consistenza interna insufficiente $(\alpha=0,19 ; 13,42 \%$ di varianza spiegata). Di conseguenza, è stata proposta una nuova analisi, considerando una struttura mono-fattoriale dello strumento, eliminando gli items 2,9, la quale ha mostrato una buona consistenza interna ( $\alpha=0,79 ; 44 \%$ di varianza spiegata). Tutti gli items considerati in quest'ultima soluzione fattoriale hanno mostrato delle relazioni robuste con la dimensione latente (comprese tra 0,55 e 0,77), di cui gli items $3,4,7$ sembrano essere i più rappresentativi. Nel dettaglio, gli items precedentemente citati fanno riferimento alla messa in atto di comportamenti legati al gioco d'azzardo nel passato $(0,72)$ e nel periodo attuale $(0,77)$ e a una delle due caratteristiche nucleari del gioco d'azzardo patologico relativa alla necessità di tenere nascosta l'entità delle loro condotte $(0,70)$. In conclusione, è possibile ipotizzare come gli items precedentemente menzionati possano essere indicativi della severità delle condotte di gioco d'azzardo nella popolazione esaminata. Per contro, gli items 2,9, che descrivono rispettivamente la convinzione che il gioco d'azzardo possa portare una marcata disfunzionalità nella vita dell'individuo e la conoscenza relativa alla diffusione di tali condotte nella popolazione di appartenenza, si ritiene siano dei fattori indipendenti tra di loro e dalla severità delle condotte di gioco d'azzardo.

Vengono più sotto riportati (Tabella 1 ) i dati relativi alle risposte dicotomiche del SPGQ, inclusi i dati mancanti; per quanto riguarda le risposte affermative, viene inoltre presentata la distribuzione suddivisa per sesso con relativo test di associazione.

Circa la metà del campione ritiene di conoscere le caratteristiche del gioco d'azzardo e, tra questi, la maggioranza sono uomi-

Tab. 1 - Frequenza di risposta agli items dicotomici; frequenza suddivisa per sesso delle risposte affermative e relativo test del $\chi^{2}$

\begin{tabular}{|c|c|c|c|c|c|c|c|}
\hline Item & Quesiti SPGQ & $\begin{array}{c}\text { Missing } \\
\text { N. } \\
(\%)\end{array}$ & $\begin{array}{l}N O \\
N . \\
(\%)\end{array}$ & $\begin{array}{l}S I \\
N . \\
(\%)\end{array}$ & $\begin{array}{c}\text { SI } \\
\text { Donne } \\
(\%)\end{array}$ & $\begin{array}{c}\text { SI } \\
\text { Uomini } \\
(\%)\end{array}$ & $\chi^{2}$ \\
\hline 1 & $\begin{array}{l}\text { Conosce le } \\
\text { caratteristiche } \\
\text { del gioco d'azzardo? }\end{array}$ & $\begin{array}{c}12 \\
(3,5 \%)\end{array}$ & $\begin{array}{c}164 \\
(48,1 \%)\end{array}$ & $\begin{array}{c}177 \\
(51,9 \%)\end{array}$ & $45,7 \%$ & $58,3 \%$ & $5,48^{*}$ \\
\hline 2 & $\begin{array}{l}\text { Ritiene possa diventare } \\
\text { una malattia? }\end{array}$ & $\begin{array}{c}13 \\
(3,8 \%)\end{array}$ & $\begin{array}{c}49 \\
(14,4 \%)\end{array}$ & $\begin{array}{c}291 \\
(85,6 \%)\end{array}$ & $86,6 \%$ & $84,5 \%$ & $n s$ \\
\hline 3 & $\begin{array}{l}\text { Ha mai giocato } \\
\text { d'azzardo puntando } \\
\text { soldi? }\end{array}$ & $\begin{array}{c}10 \\
(2,8)\end{array}$ & $\begin{array}{c}193 \\
(56,7 \%)\end{array}$ & $\begin{array}{c}150 \\
(43,7 \%)\end{array}$ & $38,5 \%$ & $49,1 \%$ & $3,92 *$ \\
\hline 4. & $\begin{array}{l}\text { Attualmente gioca } \\
\text { per denaro? }\end{array}$ & $\begin{array}{c}11 \\
(3,1 \%)\end{array}$ & $\begin{array}{c}266 \\
(77,8 \%)\end{array}$ & $\begin{array}{c}76 \\
(22,2 \%)\end{array}$ & $15,5 \%$ & $29,2 \%$ & $9,21^{*}$ \\
\hline 5 & $\begin{array}{l}\text { Gioca più di una volta } \\
\text { alla settimana? }\end{array}$ & $\begin{array}{c}15 \\
(4,2 \%)\end{array}$ & $\begin{array}{c}299 \\
(88,5 \%)\end{array}$ & $\begin{array}{c}39 \\
(11,5 \%)\end{array}$ & $8,1 \%$ & $19,2 \%$ & $3,96^{*}$ \\
\hline 6 & $\begin{array}{l}\text { Ha mai sentito } \\
\text { l'impulso a giocare } \\
\text { somme di denaro } \\
\text { sempre maggiori? }\end{array}$ & $\begin{array}{c}15 \\
(4,2 \%)\end{array}$ & $\begin{array}{c}317 \\
(93,8 \%)\end{array}$ & $\begin{array}{c}21 \\
(6,2 \%)\end{array}$ & $5,3 \%$ & $7,1 \%$ & $n s$ \\
\hline 7 & $\begin{array}{l}\text { Si è sentito di dover } \\
\text { tenere nascosta l'entità } \\
\text { del suo giocare } \\
\text { a coloro che le stanno } \\
\text { più vicino? }\end{array}$ & $\begin{array}{c}27 \\
(7,6 \%)\end{array}$ & $\begin{array}{c}299 \\
(91,7 \%)\end{array}$ & $\begin{array}{c}27 \\
(8,3 \%)\end{array}$ & $5,5 \%$ & $11,1 \%$ & $n s$ \\
\hline 8 & $\begin{array}{l}\text { Conosce persone } \\
\text { anziane che giocano } \\
\text { d'azzardo, secondo lei, } \\
\text { in modo eccessivo? }\end{array}$ & $\begin{array}{c}16 \\
(4,5 \%)\end{array}$ & $\begin{array}{c}224 \\
(66,5 \%)\end{array}$ & $\begin{array}{c}113 \\
(33,5 \%)\end{array}$ & $27,1 \%$ & $40,1 \%$ & $6,44^{*}$ \\
\hline 9 & $\begin{array}{l}\text { Ritiene che il fenomeno } \\
\text { del gioco d'azzardo } \\
\text { riguardi le persone } \\
\text { con più di } 65 \text { anni } \\
\text { di età? }\end{array}$ & $\begin{array}{c}18 \\
(5,1 \%)\end{array}$ & $\begin{array}{c}172 \\
(51,3 \%)\end{array}$ & $\begin{array}{c}163 \\
(48,6 \%)\end{array}$ & $52,1 \%$ & $45,2 \%$ & ns \\
\hline
\end{tabular}


Tab. 2 - Tipologia di giochi d'azzardo giocati nell'arco della vita e nell'attualità e distribuzione per genere

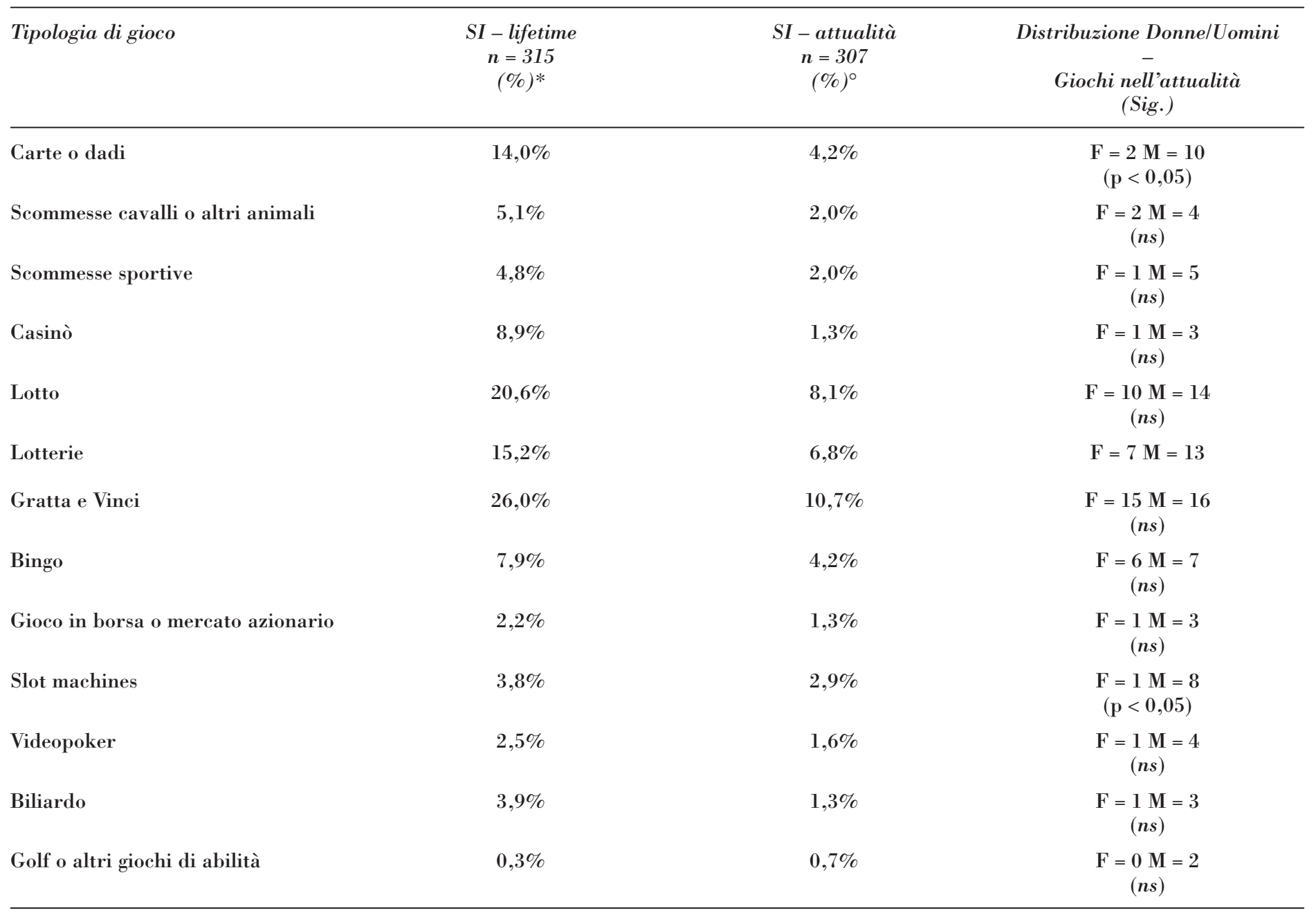

$* \mathbf{n}=45$ missing $(\%$ calcolate su $\mathrm{n}=315) ;{ }^{\circ} \mathrm{n}=46$ missing $(\%$ calcolate $\mathrm{su} \mathbf{n}=307)$

ni $(58,3 \%)$. La convinzione che il gioco d'azzardo possa diventare una malattia appartiene a più dell' $85 \%$ degli intervistati ed in questo caso non emergono differenze statisticamente significative in base al sesso. II 43,7\% del campione afferma di aver giocato d'azzardo nella vita, con una frequenza più alta tra gli uomini, statisticamente significativa; la frequenza si riduce molto rispetto al quesito precedente quando viene chiesto all'intervistato se gioca d'azzardo attualmente, quantificandosi nel $22,2 \%$ : anche in questo caso è forte lo sbilanciamento a favore degli uomini. Stesso andamento per il quesito che indaga la frequenza con cui si gioca: delle 39 persone che affermano di giocare più di una volta alla settimana, I' $8,1 \%$ è composto da donne e più del doppio sono uomini $(19,2 \%)$. Il 6,2\% del campione conferma di aver sentito l'impulso a giocare cifre sempre maggiori nel tempo, e tra questi è maggiore la frequenza tra gli uomini, statisticamente significativa. Nessuna differenza statistica emerge tra uomini e donne, invece, per quanto riguarda la tendenza a nascondere I'entità delle cifre giocate, esperienza che viene confermata dall' $8,3 \%$ del campione. La conoscenza di persone che potrebbero avere un problema di gioco d'azzardo è rilevata dal 33,5\% del campione e su questo sono più gli uomini ad avere risposto affermativamente (40,1\%). Infine, circa la metà del campione $(51,3 \%)$ non ritiene che il fenomeno del gioco d'azzardo riguardi gli over 65 anni.

Nella Tabella che segue (Tabella 2) vengono ora mostrate le frequenze di risposta agli item $3 \mathrm{~b}$ e $4 \mathrm{~b}$, i quali indagano rispettiva- mente le tipologie di gioco che la persona ha praticato nell'arco della vita e i giochi d'azzardo che riferisce di giocare nell'attualità e in ultimo la distribuzione per genere.

Vengono ora descritti e presentati i dati relativi alla frequenza con cui il campione di anziani gioca, sia nella totalità sia suddivisi per sesso (Tabella 3).

Il numero di persone che ha risposto affermativamente all'item 4 ("Attualmente gioca per denaro?") è stato di 76 su un totale di 352 (pari al 22,2\% del totale); tra questi, coloro che hanno risposto affermativamente anche all'item 5 ("Gioca più di una volta alla settimana?") sono stati 39 , pari al 50\% di coloro che dichiarano di giocare attualmente e pari all' $11,1 \%$ dell'intero campione.

Scendendo ulteriormente nell'approfondimento della frequenza di gioco alla settimana (item 5b - "Quante volte gioca alla settimana?"), in 29 hanno risposto alla domanda. In 10, pari al $25,6 \%$ di coloro che hanno dichiarato che giocano più di una volta alla settimana, non rispondono a questo quesito di approfondimento.

Vengono ora presentate più sotto le frequenze di risposta positiva all'item 6 (Tabella 4) e all'item 7 (Tabella 5), esclusivamente riferite a coloro che in precedenza hanno risposto Sì all'item 4 ("Attualmente gioca per denaro?"). A questi quesiti è stata dedicata particolare attenzione dal momento che I'aumento dell'impulso al gioco e la tendenza a mentire sono due caratteristiche peculiari del gioco d'azzardo problematico. 
Tab. 3 - Frequenza settimanale di gioco d'azzardo suddivisa per sesso e relativo test del ${ }^{-2}$

\begin{tabular}{|c|c|c|c|c|}
\hline "Gioca più di una volta alla settimana?" & $\begin{array}{c}\text { N. totali } \\
(\%) \\
(\operatorname{su~} N=338)\end{array}$ & $\begin{array}{c}\text { N. donne } \\
(\%)\end{array}$ & $\begin{array}{l}N . \underset{(\%)}{\text { uomini }} \\
\text {. }\end{array}$ & $\chi^{2}$ \\
\hline "Quante volte gioca alla settimana?" & $\begin{array}{c}\text { N. totali } \\
(\%)\end{array}$ & $\begin{array}{c}\text { N. donne } \\
(\%)\end{array}$ & $\begin{array}{l}\text { N. uomini } \\
(\%)\end{array}$ & \\
\hline Più di tre volte alla settimana & $\begin{array}{c}11 \\
(37,9 \%)\end{array}$ & $\begin{array}{c}4 \\
(36,3 \%)\end{array}$ & $\begin{array}{c}7 \\
(63,7 \%)\end{array}$ & $n s$ \\
\hline Totale & $\begin{array}{c}29 \\
(100 \%)\end{array}$ & 8 & 21 & \\
\hline
\end{tabular}

Tab. 4 - Frequenza di risposte affermative all'impulso di giocare sempre più tra le persone che giocano d'azzardo nell'attualità

\begin{tabular}{|c|c|c|}
\hline Item 4 & $\begin{array}{l}N . S I \\
(\%)\end{array}$ & $\begin{array}{c}\text { "Ha mai sentito l'impulso a giocare somme } \\
\text { di denaro sempre maggiori?" }\end{array}$ \\
\hline "Attualmente gioca per denaro?" & $\begin{array}{c}75 \\
(21,6 \%)\end{array}$ & $\begin{array}{l}\text { SI } 15(20,0 \%) \\
\text { NO } 60(80,0 \%)\end{array}$ \\
\hline
\end{tabular}

Tab. 5 - Frequenza di risposte affermative alla tendenza a mentire sul gioco tra le persone che giocano d'azzardo nell'attualità

\begin{tabular}{lcc}
\hline Item 4 & N SI & "Si è sentito di dover tenere nascosta l'entità \\
& $(\%)$ & del suo giocare a coloro che le stanno più vicino?" \\
\hline "Attualmente gioca per denaro?" & 72 & SI $20(27,8 \%)$ \\
& $(21,4 \%)$ & No $52(72,2 \%)$ \\
\hline
\end{tabular}

Tab. 6 - Frequenza di risposte affermative all'item 6 e all'item 7, suddivisa per sesso con relativo test del $\chi^{2}$

\begin{tabular}{|c|c|c|c|c|}
\hline & $\begin{array}{c}\text { SI } \\
\text { totale } \\
(\%)^{*}\end{array}$ & $\begin{array}{c}\text { SI } \\
\text { uomini }\end{array}$ & $\begin{array}{c}\text { SI } \\
\text { donne } \\
(\%)\end{array}$ & $\chi^{2}$ \\
\hline
\end{tabular}

$* \mathrm{~N}=14$ missing $(\%$ calcolate sul campione totale, $\mathrm{N}=339)$

Esplorando la frequenza di risposte affermative sia all'item 6 che all'item 7, è risultato che sul totale del campione $(\mathrm{N}=353$ persone), tra le persone che hanno fornito entrambe le risposte agli item ( $\mathrm{N}=339), 15$ di queste (pari al 4,4\% dei rispondenti) riferivano sia di aver sentito l'impulso a giocare sempre più, sia di aver mentito circa l'entità del loro giocare (Tabella 6). Tra queste, poco più della metà $(\mathrm{N}=8)$ erano uomini e non si sono rilevate differenze statisticamente significative in merito al genere.

\section{Discussione}

Alcuni studi internazionali recenti hanno dedicato più attenzione al fenomeno del gioco d'azzardo tra gli anziani: una review sulla letteratura esistente (Subramaniam, 2015) fa emergere la difficoltà di confrontare i pochi dati esistenti a riguardo, un altro studio si è focalizzato su confronti culturali tra i giocatori anziani (Costa Medeiros, 2015), un altro ancora ha cercato di individuare caratteristiche psicologiche o sociali che 
appartenevano in modo specifico ai giocatori anziani (Botterill, 2015).

Anche in Italia I'argomento sta destando più interesse rispetto al passato ed è stata condotta negli ultimi anni qualche ricerca, ma è necessario un ampliamento degli studi scientifici che possano favorire una adeguata e attendibile osservazione che sia rappresentativa della realtà nazionale.

Questo studio è nato con l'intento di approfondire la diffusione del fenomeno del gioco d'azzardo tra gli over 65 anni, di saggiare la consapevolezza degli anziani a proposito dei rischi ad esso connessi e di stimare la dimensione delle condotte di gioco problematiche o patologiche tra i giocatori anziani. Per raggiungere queste finalità è stato creato il Senior Problem Gambling Questionnaire (SPGQ) che, con pochi quesiti, consente di effettuare una valutazione del fenomeno del gioco d'azzardo in diverse direzioni: da una parte mira a individuare il livello di conoscenza di base da parte degli anziani rispetto a che cosa possa essere considerato gioco d'azzardo, dall'altro indaga la diffusione delle condotte di gioco d'azzardo nell'arco della vita e nell'attualità; consente anche di approfondire i cardini degli elementi patologici della condotta di gioco, ad esempio per quanto riguarda una alta frequenza di gioco, ma anche l'aumento dell'impulso a giocare e la tendenza a negare l'effettiva entità del proprio giocare d'azzardo; infine permette di esplorare il modo in cui gli anziani considerano il fenomeno tra i loro coetanei, con attenzione a rilevare se conoscano persone anziane che giochino eccessivamente e se ritengano che l'età dai 65 anni in poi possa essere considerata a rischio per problemi di gioco d'azzardo.

L'intenzione degli operatori, oltre a raccogliere i dati per comprendere il rischio relativo alla patologia da gioco d'azzardo di questa popolazione sul territorio, era di convogliare il pensiero degli anziani sul tema e sulla diffusione del fenomeno tra i conoscenti e i parenti per stimolare la consapevolezza circa i possibili sviluppi di gravità di alcune situazioni personali.

La ricerca è stata condotta sul territorio di Milano e provincia, con una predilezione per i luoghi di aggregazione per anziani, dove essi svolgono attività varie di tipo ludico, formativo e ricreativo. Questi centri sono gestiti prevalentemente da enti pubblici o da associazioni del privato sociale.

Il campione totale è risultato composto da 353 persone, bilanciato per sesso e con un'età media di 75,9 anni.

Nonostante la parziale consapevolezza di che cosa sia il gioco d'azzardo (come indicato dal 51,9\% delle persone che risponde in modo affermativo al primo quesito), la maggioranza del campione $(85,6 \%)$ ritiene che possa diventare una malattia, considerandone quindi i rischi correlati.

Interessante anche quanto emerge dall'item che indaga in senso generale se una persona ha mai giocato d'azzardo: circa la metà del campione risponde affermativamente. A questo proposito va specificato che in molti casi le persone hanno risposto "no" a questo item, compilando poi l'item 3b, che indaga il tipo di giochi d'azzardo praticati. Questo rafforza ulteriormente I'impressione di una non adeguata comprensione del fatto che i anche giochi legali, cioè controllati dallo Stato, quali per esempio Bingo, Gratta e Vinci, Lotto, slot-machines, siano comunque a tutti gli effetti giochi d'azzardo.

Quasi 1 persona su $4(22,2 \%)$ riferisce di giocare attualmente d'azzardo e più di 1 persona su 10 (11,5\%) afferma di giocare più di una volta alla settimana.

L'esperienza dell'impulsività nella condotta di gioco e della tendenza a minimizzare con gli altri il proprio comportamento sono ritenuti elementi essenziali e potenzialmente suggestivi di una modalità problematica o patologica nel giocare d'azzardo. II noto questionario Lie/Bet (Johnson, 1997) è composto da due quesiti che indagano proprio queste due dimensioni ed una sola risposta affermativa è ritenuta indicatore di un rischio di gioco d'azzardo problematico e meritevole di ulteriori approfondimenti; anche il questionario diagnostico SOGS (Capitanucci, 2004; Lesieur, 1987) include due domande che indagano la spinta a continuare a giocare d'azzardo senza riuscire a smettere ed il nascondimento del proprio giocare a persone importanti nella vita dell'individuo. Nel questionario SPGQ i due quesiti che, oltre alla frequenza di gioco, indagano questi possibili segnali d'allarme rispetto alla condotta di gioco sono l'item 6 e I'item 7, che chiedono rispettivamente alla persona se ha mai sentito l'impulso a giocare somme di denaro sempre maggiori e se ha sentito di dover tenere nascosta l'entità del giocare alle persone più vicine. Dai dati è emerso che il $6,2 \%$ del campione, ovvero 21 persone, riferisce di aver sentito l'impulso di giocare somme di denaro sempre maggiori; I' $8,3 \%$, corrispondente a 27 persone, afferma invece di aver tenuto nascosta l'entità del loro gioco. A questo riguardo quindi, si ritiene importante il dato che ha valutato quante persone avessero risposto affermativamente ad entrambi questi quesiti: il 4,4\% del campione totale riferisce entrambe le esperienze, il che potrebbe farci supporre che questa sia la proporzione di anziani ad elevato rischio di condotta di gioco d'azzardo patologico.

Interessante anche il dato che si focalizza sulla esperienza diretta di conoscenza di persone che potrebbero mostrare condotte di gioco d'azzardo patologico: una persona su 3 riferisce di conoscerne.

Il numero di persone che riferiscono di aver giocato nella vita è, come era immaginabile, decisamente superiore al numero di persone che riferisce di giocare attualmente: riassumendo in termini generali è possibile affermare dai dati che su 353 persone che compongono il campione, poco meno della metà ha giocato nell'arco della vita, e quasi un quarto gioca attualmente.

Dopo aver effettuato una valutazione generale di quanto emerso a proposito degli item dicotomici, si è ritenuto importante esplorare le eventuali differenze di genere: queste sono un fattore notoriamente indagato nelle ricerche cliniche sulle dipendenze e spesso si rilevano un elemento importante per tracciare profili distinti rispetto ad esempio alla gravità psicopatologica, alla modalità con cui uomini e donne affrontano il trattamento per la dipendenza e all'esito del trattamento stesso (Cano Medeiros, 2017; Kim, 2016; Sherman, 2016).

Interessanti i dati emersi a questo proposito che segnalano una omogeneità di risposta tra uomini e donne: per quanto riguarda la convinzione che esso possa diventare una malattia, l'esperienza personale di aver nascosto agli altri la reale entità del proprio giocare d'azzardo e la convinzione che il fenomeno possa riguardare gli over 65 anni. Invece, emerge una significativa disomogeneità per quanto riguarda altri elementi: gli uomini infatti mostrano più conoscenza delle donne su che cosa sia il gioco d'azzardo, hanno giocato e giocano d'azzardo più delle donne, sono giocatori più assidui; con una frequenza lievemente più alta delle donne hanno sentito l'impulso a giocare sempre più e, in ultimo, hanno conoscenza diretta più delle donne di persone che potrebbero avere problemi di gioco d'azzardo.

Per quanto riguarda l'indagine della frequenza di gioco, tra coloro che hanno affermato di giocare più di una volta alla settimana (cioè 39 persone su 353), 10 persone non hanno successivamente risposto al quesito che chiedeva quante volte alla settimana giocassero, il che potrebbe suggerire una certa resistenza all'ulteriore approfondimento della loro condotta (unitamente al fatto che questo item è quello con la maggior frequenza di missing). Delle 29 persone che hanno risposto al quesito, 18 persone (pari al $62,1 \%$ del sottocampione) hanno dichiarato di gioca- 
re da due a tre volte alla settimana, 11 persone (pari al 37,9\%) hanno dichiarato di giocare più di tre volte alla settimana. Questo dato, rilevato sull'intero campione di 353 rispondenti, indica che il 3,2\% mostra una alta frequenza di gioco. In aggiunta, considerando la relazione significativa esistente tra le risposte affermative ad almeno uno dei due item indicatori di aspetti patologici del gioco d'azzardo e la frequenza della messa in atto dei comportamenti di gioco d'azzardo $\left(\chi^{2}(2)=42,15\right.$; $p<$ $0,001)$, è possibile ipotizzare come tale porzione del campione possa essere a rischio di sviluppo di condotte di severità clinicamente significativa. Tale dato sembra in linea con i risultati precedentemente mostrati rispetto agli item considerati diagnostici. Interessante anche in questo caso soffermarsi sulle differenti risposte tra uomini e donne.

Sebbene tra coloro che riferiscono di giocare più di una volta alla settimana i due terzi siano uomini (pari al 64,1\% dei 39 rispondenti), quando si indaga la precisa frequenza con cui giocano d'azzardo, i dati indicano che uomini e donne non mostrano alcuna differenza statisticamente significativa: ovvero, all'aumentare della frequenza di gioco sembra affievolirsi l'impatto della differenza di genere. Tale conclusione sembra essere supportata dall'omogeneità della distribuzione del genere all'interno delle combinazioni di risposte che indicano un elevato rischio di gioco d'azzardo patologico. Sembra interessante focalizzarsi su uomini e donne separatamente: infatti, sebbene le donne che giocano più di una volta alla settimana siano numericamente inferiori agli uomini (14 contro 25), quando la frequenza è indagata in modo più specifico, i dati mostrano che la metà delle rispondenti (4 donne) gioca da due a tre volte a settimana e l'altra metà (4 donne) gioca più di tre volte la settimana; questo non avviene per gli uomini, tra i quali due terzi (14 uomini) dichiarano di giocare da due a tre volte a settimana e solo un terzo (7 uomini) più di tre volte a settimana. Sebbene l'item 5 che indaga la frequenza del gioco d'azzardo in senso generale presenti un elevato numero di missing, così come gli item relativi alle differenti frequenze di gioco, le mancate risposte si sono dimostrate equamente differenti equamente distribuite tra $\mathrm{i}$ sessi (item 5: $\chi^{2}(1)=0,23$; ns; item 5b: $\chi^{2}(2)=0,97$; ns). Di conseguenza, è possibile escludere possibili effetti dei dati mancanti sui risultati precedentemente discussi

I dati poc'anzi descritti potrebbero supportare un'ipotesi preliminare relativa a due differenti profili di giocatori d'azzardo in relazione al genere. Nello specifico, la tendenza al gioco d'azzardo negli over 65 anni sembrerebbe più marcata negli uomini, seppur la frequenza di tali comportamenti sembra essere moderata all'interno della settimana. Per contro, le donne mostrano una minor abitudine al gioco d'azzardo rispetto agli uomini in senso generale. Ciò nonostante, le donne che giocano d'azzardo sembrano evidenziare un'elevata frequenza di tali condotte all'interno della settimana. Tale ipotesi è in linea con i risultati di Tavares e collaboratori (2001), i quali hanno dimostrato come, differentemente da altre forme di addiction, le donne con una diagnosi di gioco d'azzardo patologico siano caratterizzate da una minor capacità di mantenere l'astensione da tali condotte rispetto agli uomini, così l'ingravescenza del disturbo è due volte più rapida nel genere femminile. In aggiunta, l'assenza di differenza significative tra uomini e donne quando questi vengono confrontati sulla frequenza di gioco d'azzardo da 2 a 3 volte alla settimana è consistente con i risultati di lbáñez e colleghi (2003) i quali hanno dimostrato come la severità del GAP sia la medesima tra i sessi. In conclusione, in linea con quanto mostrato e con I'assenza di differenze significative agli items relativi agli aspetti nucleari del gioco d'azzardo problematico, è possibile ipotizzare come nella popolazione over 65 anni, seppur gli uomini abbiamo una maggior inclinazione al gioco d'azzardo, è possibile che le condot- te problematiche associate a quest'ultimo siano indipendenti dal genere e colpiscano con la medesima severità uomini e donne. Ad ogni modo, tale ipotesi dovrebbe essere supportata da studi empirici futuri che indaghino in modo specifico tale aspetto nella popolazione di interesse.

Per quanto riguarda la propensione alla scelta della tipologia di gioco d'azzardo, si ritrovano interessanti dati sia per quanto riguarda le differenze tra il passato ed il presente, sia per quanto riguarda le differenze di genere. I giochi d'azzardo che nelI'arco della vita sono state prediletti dalle persone che hanno compilato il SPGQ sono rispettivamente i Gratta e Vinci, il Lotto e le lotterie, con frequenze riferite che variano tra il $26 \%$ ed il $15 \%$. Focalizzandosi sull'attualità, il gioco d'azzardo più diffuso risulta essere quello dei Gratta \& Vinci (con frequenza vicina all'11\%), in linea con quanto rilevato da Beato e colleghi (2015) sulla popolazione anziana bergamasca (sebbene la frequenza di gioco nell'ultimo anno ai Gratta \& Vinci sia risultata notevolmente superiore). Tra uomini e donne poche sono le differenze nelle tipologie di gioco: solo le carte o i dadi e le slot machines vengono giocati più frequentemente dagli uomini, rispettivamente con un rapporto F:M di 1:5 ed 1:8.

Questo progetto di ricerca si poneva tra gli obiettivi non solo quello di esplorare le abitudini di gioco d'azzardo ed il livello di conoscenza del fenomeno da parte della popolazione anziana del territorio lombardo, ma anche di indagare la diffusione di comportamenti di gioco che potrebbero essere considerati problematici o patologici.

Quanti anziani potrebbero essere considerati a rischio di gioco problematico?

Per rispondere a questo quesito si è tenuto presente soltanto il sottocampione di coloro che attualmente giocano d'azzardo. Ricordiamo essere circa il $22 \%$ dell'intero campione, pari a 1 anziano su 5: di questi, 1 anziano su 5 riconosce di aver provato l'impulso a giocare somme di denaro sempre maggiori e quasi 1 su 3 riferisce di aver nascosto a persone significative la reale entità del proprio giocare d'azzardo. Sempre tra coloro che oggi giocano d'azzardo, è stato interessante anche osservare quanti hanno risposto affermativamente ad entrambi gli item in questione, il che potrebbe suggerire che la condotta di gioco possa avere assunto o avere attualmente risvolti patologici: 4 anziani su 100 risulterebbero rientrare in questo quadro.

Entrambi questi dati sono da considerarsi ancora più apprezzabili per il fatto che derivano da questionari autosomministrati, il che fa ipotizzare una sottostima del dato effettivo legata innanzitutto al fenomeno della desiderabilità sociale (Baer 2003) ma, in questo caso, anche legata alle possibili difficoltà che le persone anziane possono aver avuto nella comprensione e compilazione del questionario (sebbene questa sia stata svolta appositamente alla presenza degli operatori della Task Force GAP).

In conclusione, i dati discussi nelle battute precedenti suggeriscono come il fenomeno del gioco d'azzardo sia ben noto all'interno della popolazione con più di 65 anni, con particolare riferimento alle sue caratteristiche e ai possibili rischi ad esso associati. In linea con Guiducci (2013) e Beato (2015), gli anziani sembrano prediligere giochi con basso carico cognitivo quali Gratta e Vinci, Lotto e Lotterie. Inoltre, seppur il fenomeno del gioco d'azzardo sembrerebbe essere maggiormente praticato dagli uomini, tale differenza non sembra mantenersi all'interno del sottogruppo dei giocatori assidui, all'interno del quale il genere si distribuisce in modo omogeneo. Infine, I'assenza di differenze di genere per quanto concerne le caratteristiche nucleari del disturbo da gioco d'azzardo e la rilevante porzione di soggetti che dichiara di aver riconosciuto tali elementi, supportano studi futuri volti all'indagine di elementi di 
rischio o patologici all'interno della popolazione over 65 anni mediante la somministrazione di interviste cliniche volte alla valutazione approfondita della severità della condotta, congiuntamente alla chiarificazione dell'esordio, del decorso e dei fattori precipitanti.

Tenendo presente il continuum tra non giocatori d'azzardo, giocatori non problematici e giocatori problematici, da questo studio sembra emergere che:

- il fenomeno del gioco d'azzardo riguarda una parte limitata della popolazione over 65 anni, che, se gioca d'azzardo, predilige giochi a basso investimento cognitivo;

- il fenomeno del gioco d'azzardo ha una maggior diffusione tra gli uomini che mostrano complessivamente una esperienza personale e indiretta maggiore delle donne;

- in una porzione esigua di coloro che giocano, l'abitudine a giocare d'azzardo perde la valenza ricreativa e assume connotazioni problematiche (rilevate attraverso indicatori quali una alta frequenza di gioco, l'intensificazione dell'impulso a giocare quantità maggiori di denaro, la tendenza a mentire sulle somme giocate);

- seppur il gioco d'azzardo all'interno della popolazione over 65 sembra essere maggiormente;

- praticato dagli uomini, sembra emergere una preliminare evidenza circa un'equidistribuzione delle caratteristiche problematiche del gioco d'azzardo tra i sessi.

Detto ciò, è utile menzionare alcuni limiti che hanno caratterizzato il presente studio, i quali sembrano essere di utilità per approfondire il tema discusso con studi futuri. Nello specifico, i risultati mostrati potrebbero risentire degli effetti del contesto all'interno del quale la ricerca è stata condotta. Nel dettaglio, dato che la somministrazione dello strumento è stata effettuata durante le azioni di sensibilizzazione relative al gioco d'azzardo all'interno della popolazione anziana, i dati riguardanti la consapevolezza dei rischi associati a tali condotte potrebbero essere sovrastimati. Di conseguenza, per sopperire a tali problematicità, i risultati presentati dovranno essere replicati riproponendo le medesime valutazioni utilizzando dei campionamenti randomizzati all'interno della popolazione generale over 65 anni. Un altro aspetto di criticità fa riferimento allo strumento utilizzato. Seppur il SPGQ si è dimostrato uno strumento di facile somministrazione al fine di raccogliere informazioni su un largo campione, la struttura fattoriale non ha supportato l'ipotesi iniziale relativa a due dimensioni connesse con la conoscenza/consapevolezza del gioco d'azzardo e la rilevazione della messa in atto di condotte di gioco d'azzardo. Per contro, lo strumento ha mostrato delle proprietà psicometriche adeguate quando venivano eliminati due items $(2,9)$, descrivendo in tal senso un'unica dimensione relativa alla severità delle condotte di gioco d'azzardo. Di conseguenza, sono necessari studi futuri volti da un lato a replicare la robustezza psicometrica dello strumento nella sua nuova forma su diversi campioni, e dall'altro a dimostrare la validità esterna dello strumento, nello specifico valutando le relazioni esistenti tra il SPGQ ed altri questionari che valutano la severità del gioco d'azzardo nella medesima popolazione, così come la sua validità predittiva rispetto alla diagnosi di disturbo da gioco d'azzardo secondo i criteri del DSM-5 su soggetti over 65 anni.

\section{Bibliografia}

Alberghetti A., Collins P. (2015). A passion for gambling: a generationspecific conceptual analysis and review of gambling among older adults in Canada. Journal of Gambling Studies, 31: 343-358. DOI: 10.1007/s10899-013-9425-2.
Baer R.A., Rinaldo J.C., Berry D.T.R. (2003). Self-report distortions (including faking, lying, malingering, social desirability). Encyclopedia of psychological assessment, 2: 861-866. DOI: 10.4135/9780857025753.n181.

Beato E., Biffi L., Marchesi L., Riglietta M., Gori M., Potente R., Molinaro S. (2015). Anziani e gioco d'azzardo: dai dati alle azioni. In: Lucchini A. (a cura di). Il gioco d'azzardo patologico. Milano: FrancoAngeli.

Botterill E., Gill P.R., Mc Laren S., Gomez R. (2016). Marital status and problem gambling among Australian older adults: the mediating role of loneliness. Journal of Gambling Studies, 32(3): 1027-38. DOI: 10.1007/s10899-015-9575-5.

Cano M.Á., De Dios M.A., Fernández V.C., Childress S., Abrams J.L., Roncancio A.M. (2017). Depressive symptom domains and alcohol use severity among Hispanic emerging adults: Examining moderating effects of gender. Addictive Behaviors, 27;72: 72-78. DOI: 10.1016/ j.addbeh.2017.

Capitanucci D., Carlevaro T. (2004). Guida ragionata agli strumenti diagnostici e terapeutici nel disturbo di gioco d'azzardo patologico. Disponibile on line al sito: www.andinrete.it/portale/documenti/pdf/ manuale_strumenti_diagnostici_gap.pdf.

Costa Medeiros G., Leppink E., Yaemi A., Mariani M., Tavares H., Grant J. (2015). Gambling disorder in older adults: a cross-cultural prospective. Comprehensive Psychiatry, 58: 116-121. DOI: 10.1016/j.comppsych.2014.12.021.

Decreto Balduzzi. DL. 13 settembre 2012, n. 158 - L. 8.11.2012, n. 189. Disposizioni urgenti per promuovere lo sviluppo del Paese mediante un più alto livello di tutela della salute.

Desai R.A. (2004). Anziani. In: Clerici M. (a cura di). Il gioco d'azzardo patologico. Springer (2011). DOI: 10.1007/978-88-470-1538-8.

Guiducci M. et al., Gruppo Abele, Auser, Libera (2013). L'azzardo non è un gioco. Gioco d'azzardo legale e rischio dipendenza tra le persone over 65 incontrate da Auser. Disponibile on line. http://images.auser.it/lT/f/ img_biblioteca/img89_b.pdf.

Johnson E.E., Hammer R., Nora R.M., Tan B., Eistenstein N., Englehart C. (1997). The lie/bet questionnaire for screening pathological gamblers. Psychological Reports, 80(1): 83-88. DOI: 10.2466/pr0.1997.80.1.83.

Kim H.S., Hodgins D.C., Bellringer M., Abbott M. (2016). Gender Differences Among Helpline Callers: Prospective Study of Gambling and Psychosocial Outcomes. Journal of Gambling Studies, 32(2): 605-23. DOI: 10.1007/s10899-015-9572-8.

Ibáñez A., Blanco C., Moreryra P., Sáiz-Ruiz J. (2003). Gender differences in pathological gambling. The Journal of clinical psychiatry, 64(3): 295-301.

Lesieur H.R., Blume S. (1987). The South Oaks Gambling Screen (SOGS): a new instrument for the identification of pathological gamblers. American Journal of Psychiatry, 144(9): 1184-8. DOI: 10.1176/ajp.144.9.1184.

Mc Neilly D.P., Buke W.J. (2000). Late life gambling: the attitudes and behaviors of older adults. In: Grant J.E., Potenza M.N. II gioco d'azzardo patologico. Spinger (2011). DOI: 10.1007/978-88-470-1538-8.

Mc Neilly D.P., Buke W.J. (2002). Disposable time and disposable income: problem casino gambling behavior in older adults. Journal of Clinical Geropsychology, 8: 75-85. DOI: 10.1007/978-88-470-1538-8.

Pavalko R.M. (2002). Problem gambling among older people. In: Grant J.E., Potenza M.N. Il gioco d'azzardo patologico. Spinger (2011). DOl: 10.1007/978-88-470-1538-8.

Regione Lombardia, Legge Regionale 21 ottobre 2013 n. 8. Norme per la prevenzione e il trattamento del gioco d'azzardo patologico (BURL n. 43, suppl. del 22 ottobre 2013).

Sherman B.J., Baker N.L., McRae-Clark A.L. (2016). Gender differences in cannabis use disorder treatment: Change readiness and taking steps predict worse cannabis outcomes for women. Addictive Behaviors, 60: 197-202. DOI: 10.1016/j.addbeh.2016.04.014.

Subramianam M., Wang P., Soh P., Vaingankar J.A., Chong S.A., Browning C.J., Thomas S.A. (2015). Prevalence and determinants of gambling disorder among older adults: a systematic review. Addictive Behaviors, 41: 199-209. DOI: 10.1016/j.addbeh.2014.10.007.

Tavares H., Zilberman M.L., Beites F.J., Gentil, V. (2001). Brief communications: Gender differences in gambling progression. Journal of gambling studies, 17(2): 151-159. DOI:10.1023/A:1016620513381. 


\section{Dipartimento delle Dipendenze}

\section{Senior Problem Gambling Questionnaire - SPGQ \\ Strumento epidemiologico per popolazione over 65 anni}

(Annalisa Pistuddi, in collaborazione con GISSMA - Gruppo Italiano per lo Studio dello Stress e delle Metodiche Antistress)

Luogo dell'intervista

Data Età

\section{$\square$ Maschio $\square$ Femmina}

Stato civile:

$\square$ Nubile/celibe $\square$ Separata/o - divorziata/o $\square$ Vedova/o $\square$ Coniugata/o

\section{SEGNARE LA RISPOSTA CORRETTA CON UNA X}

1) Conosce le caratteristiche del gioco d'azzardo?

(Le caratteristiche sono tre: si puntano soldi o valori, la posta non è ritirabile e l'esito dipende dal caso)

2) Ritiene che possa diventare una malattia?

3) Ha mai giocato d'azzardo, puntando soldi?

Se sì quali giochi ha praticato?

$\square$ carte o dadi a soldi $\square$ scommesse cavalli o altri animali

$\square$ scommesse sportive $\square$ casinò $\square$ lotto $\square$ lotterie

$\square$ gratta e vinci $\square$ bingo

$\square$ gioco in borsa o mercato azionario

$\square$ slot machines $\square$ videopoker

$\square$ biliardo $\square$ golf o giochi abilità per denaro

4) Attualmente gioca per denaro?

Se sì a quali giochi?

$\square$ carte o dadi a soldi $\square$ scommesse cavalli o altri animali

$\square$ scommesse sportive $\square$ casinò $\square$ lotto $\square$ lotterie

$\square$ gratta e vinci $\square$ bingo

$\square$ gioco in borsa o mercato azionario

$\square$ slot machines $\square$ videopoker

$\square$ biliardo $\square$ golf o giochi abilità per denaro.

5) Gioca più di una volta a settimana?

Se sì, quante volte gioca?

$\square$ da due a tre volte alla settimana $\square$ più di tre volte alla settimana

6) Ha mai sentito l'impulso a giocare somme di denaro sempre maggiori?

7) Si è sentito di dover tenere nascosta l'entità del suo giocare a coloro che le stanno più vicino?

8) Conosce persone anziane che giocano d'azzardo, secondo lei, in modo eccessivo? 